\title{
Is Observation Mathematically-Laden?
}

\author{
Thomas Michael MülleR
}

\begin{abstract}
In this article, we will defend the epistemological claim that observation is mathematically-laden. A well-known thesis in the philosophy of science is that observation is theory-laden. The claim that observation is mathematically laden can be similarly justified. The first part of the paper focuses on the definition of mathematically-ladeness and its relations to the best-known problem of theory-ladeness. The second part of the paper presents some explicit examples and outlines the consequences and the difficulties of this epistemological limit. Finally, a specific context for this problem is discussed in detail: we will analyze the question of deterministic chaos as a paradigmatic example of mathematically-ladeness and show that the deterministic or indeterministic nature of chaos is strongly linked to the choice of a particular mathematical description.
\end{abstract}

Keywords: Epistemology; theory-laden; mathematically-laden; determinism; deterministic chaos.

\section{Introduction}

In recent years, a considerable amount of work has been done in the foundations of sciences and philosophy of sciences to discuss subjects such as determinism, causality (Earman 1986, Norton 2008), or pointillisme (Butterfield 2006). The main idea of these works is to analyze the mathematical structure of a given scientific theory, the form of the equations that express the laws of those theories or some possible toymodels, and then argue that determinism is true (or false) of a given theory, that causation is an illusion, that points are real physical entities, and similar ontological claims.

All these works share a common attitude: mathematics is seen as a neutral language that directly links a scientific theory to reality: if the equations of motion have a unique solution, then determinism is true, if there is more than a solution, than causality fails, and so on. The premise always relies on some mathematical feature, and the consequence involves some ontological claims.

In this article we want to show that mathematics is not a neutral instrument but, rather, it influences and shapes our understanding of reality. Mathematics is a language, but not a neutral one. Therefore, ontological commitments based solely on the analyses of mathematics should be considered carefully.

The structure of the paper is as follows: Section 2 provides a quick overview of the classic debate about the theory-ladeness of observation, and performs a comparison with the mathematically-ladeness by giving few concrete examples.

Principia 17(1): 165-181 (2013).

Published by NEL — Epistemology and Logic Research Group, Federal University of Santa Catarina (UFSC), Brazil. 
Section 3 gives a more general overview of the problem and provides some more detailed examples.

In section 4 it is argued that the notion of determinism applied to a chaotic system is controversial. We discuss the notion of deterministic chaos and show that the main ideas underlying deterministic chaos is a consequence of using a certain kind of mathematical tools.

Finally, section 5 gives some concluding remarks.

\section{Observation is theory-laden}

A well-known thesis in the epistemology of science is that observation is theoryladen. This position has been advocated by Hanson (1958), in his famous book "Patterns of discovery" which could be seen as a development of Sellars' ideas (Sellars 1997 [1956]).

The argument runs as follows: Sellars considers that empiricism is infected by the "myth of the given". The myth consists in the idea that there could be sensorial experience which is not conceptualized. But Sellars answers that, if an experience is not conceptualized, it can't be used to justify our propositions. Therefore, our sensorial experience is conceptualized, and requires a network of concepts and a learning process to acquire them. We call this network a theory or a system of beliefs. ${ }^{1}$

Acquiring knowledge via sensorial experiences can modify any system of beliefs. Nonetheless, this system of beliefs is necessary for a sensorial experience to happen. A proposition about our sensorial experience (such as "this apple is red") can be justified only by using some other propositions and, therefore, the entire system of beliefs concerning, e. g., the color of the objects is required to acquire the new knowledge about the redness of the apple. A new proposition is justified by integrating it in a system of pre-existing coherent propositions, viz. a system of beliefs.

Hanson goes further by claiming that the theory (or the system of beliefs) forms the sensorial content of our observations; ${ }^{2}$ our theory determines what we perceive and what we don't perceive. We have to learn how to look at a given problem, in order to see certain patterns. To support this claim, Hanson gives a famous example: it pictures Kepler with Tycho Brahe observing the rising sun. Hanson affirms that Tycho and Kepler can't see the same thing since Kepler has a different theory about the dynamics of the Earth and the Sun than Tycho. They both see a flat shiny disc moving in the sky, but their theoretical background transform this image into:

1. A moving body rotating around the earth for Tycho.

2. A fixed star around which the earth moves for Kepler.

Principia 17(1): 165-181 (2013). 
This argument can lead to two different conclusions, and it is unclear to which of them Hanson adheres,

I. Having a theory/system of beliefs as a conceptual background is a necessary condition for recognizing patterns which could not be observed otherwise.

II. Adherents of different theories/systems of beliefs cannot observe the same things.

This clear cutoff is well illustrated by Esfeld (2009). Claim (I) does not imply claim (II); claim (I) outlines the fact that our ability to interpret limits our ability to recognize new facts. Claim (II) is more radical and affirms that it is impossible for proponents of two different theories to convince their opponents on an objective ground. This second position seems to be false: In fact, Kepler was able to convince the scientific community of his time using arguments based on observation.

As a matter of fact some observations could be explained only by an adherent of the Keplerian theory of planetary motion, and even if Tycho never recognized the Copernican planetary system, it is possible for us to decide on an objective ground that the Earth moving around the Sun, the Sun occupying one of the foci of the ellipse, is a better approximation of the planetary motion than the geocentric one.

Therefore Hanson's claim could be understood in two ways, i.e. that

i. Our observation can't be separated in an empirical and a theoretical part (i.e. a bare sensorial experience and a conceptualization).

ii. There is no possible objective scientific consensus on a given theory.

but the two claims are distinct, and the first does not imply the second. In general a scientific realist could accept the first claim, but strongly rejects the second. The main difference between the theory-ladeness and the mathematically-ladeness will be that this cutoff criterion is untenable for the case of mathematically-ladeness.

\subsection{Is observation mathematically-laden?}

In this article we will argue that observation is not only theory-laden, but it is also mathematically-laden. This means that mathematical structures are not neutral; they can have a direct influence on our mental representation of reality. Different mathematical structures can shape our intuition on our scientific observation and measurements, they can be a guide or a handicap in the development of new theories, and, moreover, they can change our metaphysical understanding of reality. We claim that mathematics is a language, but that it is entangled with the theory that uses it: it is not possible to clearly separate the semiotic part (mathematics) and the semantic (physics, chemistry, biology, economics, or whatever else mathematics is applied 
to). This problem arises explicitly when - given a single theory - it is possible to choose between two different mathematical structures, which are coherent with the same empirical observations, but the mathematical objects in those descriptions are different.

It is possible that two mathematical formalisms that have a priori nothing in common (two different "mathematical ontologies") can be used to describe the same set of empirical data. The consequence is that those data are "seen" as something different, depending on the mathematical apparatus we use (analogously to the sun which was "seen" as something different from Kepler and from Tycho, depending on their theory).

This position is not completely new, and we could even say that it has been floating around during at least the last fifteen years, taking (slightly) different forms. As an example, the interested reader could look at Jones (1991); more recently, the possible coexistence of two mathematical structures, describing the same set of physical phenomena has been discussed in the debate concerning structural realism (Ladyman 1998). Nonetheless, it has never been put in a definite, straightforward form. Some authors discuss only very specific cases: the Heisenberg versus Schroedinger formulation of quantum mechanics is probably the most discussed topic, but also one of the more controversial (see Muller 1997a, 1997b, 1999 and Madrid-Casado 2008). The debate concerning the mathematical-ladenness of observation is also entangled with the theory-laden debate and often confusedly treated as a part of it.

The question concerning the theory-neutrality of mathematics has been discussed outside physics, and especially between economists: physicists often seem to endorse the position that mathematics is theory-neutral. Economists seem to endorse the opposite view (Israel and Ingrao 1990, Israel 1996): they take for granted that mathematics is not theory-neutral, and they discuss the influence of different mathematical structures on the development of their discipline. Of special interest is (Israel 1993), in which a historical case study is analyzed; the final choice between two kind of mathematical analysis is shown to be socially-motivated. Nonetheless some minor cases of interest exist also in physics: e.g. the discussion on the Whitehead-Synge theory of general relativity (Müller 2006, Bramè 2006) questions to some extent the influence that equations have on the subsequent ontology, and it seems that Whitehead was to some extent motivated by aesthetic reasons when writing his theory of general relativity. More recently it has been argued that mathematics may be seen as the structure of scientific theories (Madrid-Casado 2011), but that the distinction between structure and ontology has no clear boundaries, and therefore collapses: mathematical structures are ontologically-laden. We are much sympathetic to this view, that seems to be close to ours.

Unfortunately, none of those authors explicitly addresses the question of the theory-ladeness of mathematics in the precise sense that we defend. It would be 
useful to provide explicit examples of the influence of mathematics on our observations; more precisely, it would be particularly useful to show some examples in which the mathematically-ladenness of observation has important and disturbing consequences on our today conception of the world: this is the aim of the paper.

There is a fundamental difference between "theory-laden" and "mathematicallyladen": when we say that some observation is theory-laden, e.g. Tycho versus Kepler, we can hope that some new phenomena will, sooner or later, be incompatible with one theory, but compatible with the second, and therefore the first theory will be falsified, and the second will survive. Unfortunately, two mathematical structures describing the same set of empirical phenomena are indistinguishable on a purely empirical ground: this is due to the indirect link between mathematics and reality. Mathematics does not directly link to reality: it links to some physical theory, and the physical theory is linked to reality. A similar situation has been discussed for semiotic: semiotic does not directly refer to real objects, but refers to semantic, and through semantic it refers to real objects. The case is similar, but here we are claiming that mathematics refers to some theory and at the same time it shapes and influences the theory, which then (pretends to) describe some features of reality.

In our case studies, we will discuss situations where two different mathematical descriptions are related to one or, sometimes, two theories: the important point under scrutiny is that both theories and therefore both mathematical structures refer to the same set of empirical data. One should not believe that the problem of mathematically-ladeness manifests itself only when two different mathematical descriptions are present. When two mathematical description are possible, then we can identify the problem, but mathematics has an influence even when only one mathematical description exists: to some extent those cases are more concerned with mathematically-ladenness, since it is more difficult to identify the extent and the specific way in which the mathematical descriptions shapes our understanding of reality.

Before giving some examples, we would like to contextualize the specific fields of research that are potentially concerned with mathematically-ladeness to a great extent. Consider all the attempts that have been done to discuss ontology in a scientificinformed and scientific-oriented way: e.g. the debate between relationalism and substantivalism in the context of spacetime theories (Earman 1989, Friedman 1983). If spacetime really exists or not (in the sense, say, of 'absolute space'), is a matter of ontology, i.e. it is about the way our world really is. Nonetheless, the discussion on this subject is to a large amount a discussion of the mathematical form of the equations of a given theory. But then we are deducing from a linguistic form (mathematics) something about ontology (i.e. reality). Of course, people concerned with this debate will argue that relying on our best scientific theories is the best way to inquire about ontology (and as a scientific realist one could hardly disagree); they will affirm 
that what is at stake is not a matter of language, but the intimate nature of reality. Still the question will be pertinent: to what extent is this dispute a matter of linguistic expression? The reason to trust our best scientific theories is that they match with empirical facts; we feel then justified in 'asking' those theories how the world really is, and to inquire into the kind of ontological commitment they presuppose. This will grant us a 'quick look' at ontology. But our best scientific theories rely on mathematics to such an extent that it is practically impossible to express them without mathematics. In some cases, discussions about what a physical theory really says are discussions about the form of equations, the existence and unicity of solutions, the way to obtain a set of equations. ${ }^{3}$ In that sense, it is a discussion about mathematics, and the form of mathematics that we use shapes our intuition. It changes our understanding of a given theory. Finally, as it has been well explained by Hanson, it will change our conceptualization of new data, and therefore influences our observation.

We will discuss in more detail all these questions in section 4.

\section{Mathematical bias}

In order to clarify the problem we are presenting, we would like to give a list of examples. There are some well-known examples, and some that are less obvious and require special treatment. We will shortly give a few easy examples, and then move to more controversial cases. Our aim is not to defend a specific mathematical model, but to stress the fact that a mathematical model influences our understanding of reality. Our aim is, rather, to show that mathematical structures are not theory-neutral, and that the presence of two mathematical structures highlights this problem.

Consider as an example the matrix formalism of Heisenberg or the wave-function formalism of Schroedinger, which have been proven isomorphic (see Muller 1997a, 1997b, 1999 and Madrid-Casado 2008) and which equally capture the theory of quantum mechanics. In this case we have only one theory - quantum mechanics but two mathematical structures.

The choice of a given mathematical structure can influence the scientific imagination and the philosophical investigations about the meaning and the content of science. For instance, a proponent of the Schroedinger formalism will most commonly use the image of the wave-packet, an advocate of the Heisenberg matrix formalism will be pushed on a somewhat different direction. It has been claimed that despite these two formalisms being equal on the empirical level, they are not on the ontological level (Madrid-Casado 2011); especially Heisenberg's representation is discrete, while Schroedinger's is continuous. Therefore, the mathematical description of a physical reality has an influence on our intuition, and can be a constraint on our imagination when we encounter a new phenomenon.

Principia 17(1): 165-181 (2013). 
A scientific realist may think that Heisenberg's and Schroedinger's formalisms are two representations of the same physical reality: whatever formalism is used, it refers to something real. But the difficulty comes with the fact that we have to use mathematics in order to speak about this physical reality, and that the mathematical language guides our intuitions and our theory/system of beliefs about the physical reality. In a very similar way, Kepler and Tycho are guided by their respective theories when observing the rising sun. We are guided by our mathematical description when observing quantum phenomena: the difficulty is that it is not possible to find an empirical discriminator between two mathematical representations. Reality is veiled from us; we can infer only part of it, based on empirical constraints. Certainly not every mathematical difference in formalisms is a difference in ontology: it would be great to find an argument for a distinction between two mathematical structures, in order to know if it is only lexical or if it has ontological bearing. Unfortunately this can't be done, since reality is veiled, and mathematical structures are entangled with theories.

This is obviously an epistemological problem: Proponents of different mathematical structures can't "see the same things", and they can't convince their opponents on an objective ground. They can only recognize the coherence of a different mathematical formalism, its elegance or its beauty. ${ }^{4}$ Mathematics can't rely on observational criteria, as it was the case of the theory-ladenness of observation, since there is nothing to observe in the physical world that has an obvious mathematical nature, even for followers of radical Platonism.

Suppose Schroedinger decided to study philosophy instead of physics and never invented his wave-formalism. We would use the Heisenberg matrix formalism today, and none of us would be aware of the wave-packet or of similar ideas. We would probably have found the same results, we would have discovered superfluidity, superconductivity, spin resonance and so on, but we would have a different image of these phenomena, and we would tell different stories about them to our students. They in turn would have a different intuition about them. After all, in a possibleworld-without-Schroedinger, it is not sure that we would know the same things about Quantum Mechanics. Not because these facts are not real (the possible-worldwithout-Schroedinger may be a physical world identical to our own), but because none of us would even think about something like a wave-packet.

There are many interesting similar situations: For instance, the case of Newton's three laws and the Lagrangian-Hamiltonian formalism about Mechanics. If we use Newton's laws, we will think in terms of point-like masses moving on trajectories that can be calculated via differential equations. Consider what we think about the problem of the inclined plane and a ball rolling down it. Generally we think there is a first force - gravity - that is directed toward the center of the earth. A second force - reaction - pushes the moving ball perpendicularly to the plane; therefore par- 
tially, but not completely compensating the effect of gravity. The remaining gravity is the cause of the rolling down.

Now switch to a Lagrangian formalism: In the Lagrangian we have kinetic energy and potential energy. We also encounter some constant of movement, but we don't have anything like a reaction force. What about a world in which scientists can only rely on the Lagrangian formalism? They would know nothing about reaction forces; this concept would not exist for them in the same sense in which we understand it.

Another case: Bohmian's and Everett's interpretation of Quantum Mechanics. They clearly have the same observational consequences. Therefore, no one can distinguish them on an empirical, objective ground. So where's the difference? We can answer that the difference is in the idea we have of those two theories. But where does this idea come from? It comes from the mathematical model we use. Consequently, in this case, the mathematical model is the interpretation (or at least it can't be separated from the interpretation).

We should notice that our three examples have consequences on different levels. Proponents of Everett or Bohmian mechanics subscribe for different physical ontology. They believe in a world that is physically different, or at least they think so.

To show the difference, proponents of, say, standard versus non standard analysis, are defending a distinct mathematical ontologies, but they can nonetheless believe in the same physical ontology. The world in which they believe is different only if they are mathematical realists.

Finally, the case of the Lagrangian versus Newtonian physicist is a controversial one. It is unclear if a reaction force should count as a physical something, or only as a mathematical something.

We see that the problem we outline has different possible influences: on our mathematical ontology, on our physical ontology, and on the possibility to clearly distinguish between the realm of mathematics and the realm of physics.

\section{Unnoticed mathematical bias}

We could imagine some deeper questions, with profound implications for philosophers, as in the case of deterministic chaos. Deterministic chaos has been a matter of concern during the last decades, especially regarding the meaning of the designation 'deterministic'. Some authors have argued that chaos theory is to some extent incompatible with determinism, while others have focused on the difference between unpredictability (which is certainly true of chaotic systems) and indeterminism (Prigogine and Stengers 1988, Bricmont 1995). As Earman (1986) explains very well, whether the world is deterministic is an ontological question, while predictability is an epistemological one. As a consequence the debate has mainly focused on show-

Principia 17(1): 165-181 (2013). 
ing that despite the unpredictability of chaotic systems, nature as it is described by our best scientific theories is deterministic. It has been argued that determinism is to some extent more a premise than a consequence of scientific theories (Israel 1992), but the point remains that chaos can't per se dismiss determinism.

The question that arises at this point is: if empirical data look random (impredictability), why are we speaking of deterministic chaos? The deterministic pretension is linked to the form of chaotic equations: the differential equations have a unique solution, i.e. given an initial condition, they allow to compute a unique dynamic. This dynamic is very sensible to initial conditions, i.e. if one computes two very close but different initial conditions, the result will quickly diverge, and the impredictability arises from the fact that we have a limited ability to set initial conditions, and therefore we must accept that we will observe very different behaviors, because we have in fact set our experiment with slightly different initial conditions. Would one be able to reach an infinite degree of precision, he would observe absolute determinism, or at least this is what our equations of motion express. Hence nature is unpredictable but deterministic.

A similar line of argument has been disputed since at least Suppes (1993), because deterministic and indeterministic systems may in fact be indistinguishable from an empirical point of view. In a recent couple of articles, Werndl $(2009,2013)$ has argued that, under certain conditions, deterministic descriptions are observationally equivalent to stochastic (therefore indeterministic) ones. In fact, Werndl point concerns the underdetermination problem: she claims that the same set of data can fit two different theories, because a given set of data is always less rich in information than the theory that describes it. Werndl is right with this claim, but fails to address an important question: Where do the deterministic — or the indeterministic — descriptions come from? A stochastic process is just a mathematical model in which we use probabilities; if our probabilities are ontological and not epistemic (i.e. if we think that those probabilities express an objective feature of nature and not a lack of knowledge) then a stochastic process is a paradigmatic example of indeterminism. But where does the deterministic description come from?

As we said, it comes from a choice of mathematical formalism: certain kind of chaotic systems are so sensible, that even an infinitely small error in the initial condition will generate a totally different evolution in time. In this case, the deterministic nature of chaos is nothing more than a petitio principii: if one can localize two identical systems into the same Euclidean point (the idealization of an infinite precision) as an initial condition, then the two systems will behave absolutely identically. Of course this is practically unfeasible, and no one will pretend anything different. And this is precisely the nature of chaos: it is ontologically deterministic and epistemically unpredictable.

But now consider this second question: what is a Euclidean point? One would 
answer that a Euclidean point is a mathematical object: a primitive in the axiomatic of Euclid and Hilbert. Does a Euclidean point really exist? Maybe. But the sole reason to believe in the ontological existence of Euclidean points is that we use them in our best scientific representation, i.e. the most used mathematical descriptions of the physical world, require Euclidean points. Unfortunately this is clearly not enough to pretend that Euclidean points are real: there is no more reason to believe in them than in any other mathematical object.

Consider the case of a N-particle system such as a gas in a box. The deterministic description of the trajectory of the N-particle system in the phase space is an example of deterministic chaos: the deterministic commitment comes from the idea that each particle is well described by a Euclidean point, its position, and a classical trajectory, a curve on a 3 -dimensional Euclidean space. ${ }^{5}$ This idea is corroborated by the fact that we have always described particles as Euclidean points, even when everyone knows that this description is only an approximation, (as in the case of the inclined plane and ball identified with its centre of mass rolling down the plane) and that this description works, allowing us to predict the trajectory of the ball. Nonetheless, it is possible to change our description of geometry, and use a geometry in which there are no Euclidean points. We will provide a mathematical model where there are no Euclidean points. Therefore there will be no infinite precision available (on the ontological level): my conclusion is that what we consider to be ontologically true in this example depends on the mathematical formalism that we choose; nevertheless, mathematics is only a language and the ontology of the world can't depend on the subjective choice of a mathematical formalism. This would then show an example on how our theories and our observations are mathematically-laden. ${ }^{6}$

The idea works like this: The classic axiomatic of Hilbertian geometry supposes that points, lines, planes are primitives and that — given a list of axioms - it is possible to build everything else. A geometry without points can be built if we change our set of primitives. Pecoraro and Gerla (Pecoraro and Gerla 2006, Gerla 1990) for example, following on original idea of Whitehead (Whitehead 1929), have proposed to construct a geometry using regions as primitives instead of points. Suppose that we have regions of space, and the notion of "being in contact" between two regions. This is our set of primitives: We don't define "regions"7 and we don't define "being in contact". With these primitives we can build everything else.

How can we do this? Consider the picture in Figure 1; one can define four kinds of relations of connexion:

1. Let's define $C(x)$, as the set of regions that touch $x$. In this case you can say that if all regions in contact with $x$ are in contact with $y$, then $x$ is included in $y$. In this case, write $x \leqslant y$. We call this type of relation "inclusion".

2. Imagine that two regions $x$ and $y$ have a common part. This is true if a third

Principia 17(1): 165-181 (2013). 


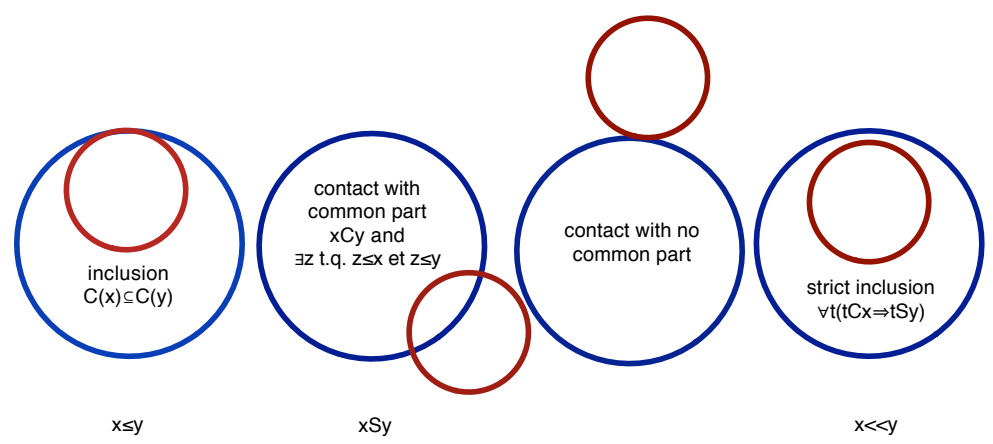

Figure 1: 4 different connexions

region which is included in both $x$ and $y$ exists. Write $x S y$ for " $x$ has a common part with $y^{\prime \prime}$.

3. Imagine that two regions are connected without any common part. You just have to deny what has been supposed on step 2 .

4. One region $x$ could strictly be included in a region $y$. This is the case if every region which is in contact with $x$ has a common part with $y$. "Having a common part with" has been defined on step 2 . We write " $x$ is strictly included in $y " x \ll y$.

Now consider a set of regions $S$ and a subset $A$ of $S$ such that

1. for every $a_{1} a_{2}$ in $A a_{1} \ll a_{2}$ or $a_{2} \ll a_{1}$ or $a_{1}=a_{2}$ (otherwise said, $A$ is a total order for $\ll)$

2. there isn't any region $s$ in $S$ such that for every $a_{i} s \ll a_{i}$ (that is, no minimal element)

We call this set of regions an "abstractive class". Abstractive classes can be shown to be equivalent to geometrical objects such as lines, planes, and especially points. In particular Pecoraro and Gerla have proved that the equivalence class of minimal abstractive classes is isomorphic to Euclidean points. This is what we wanted: We have built some objects that play the role of Euclidean points. Nonetheless only regions exist in our mathematical description. Therefore, when we solve a physical problem, we should use only regions as initial or boundary conditions (we can choose any mathematical ontology, but when the choice is done, we should be ontologically rigorous). In fact, abstractive class are a mathematical translation of the process of being 'as precise as we want' without being 'infinitely precise'.

When a region is used as an initial condition, deterministic problems in physics with a non-chaotic behavior are still deterministic. ${ }^{8}$ Consider the case of the inclined 
plane: if the image we have for the centre of mass is a point, the evolution in time follows a deterministic trajectory. If the position of the centre of mass is a small region, nothing changes. In contrast, consider the case of a chaotic system, such as a dynamical billiard (Bunimovich billiard or Sinai billiard). If the initial position is thought to be a point, then the evolution follows a deterministic trajectory, and the unpredictability of the observed behavior is explained as an extreme sensibility to initial conditions. But if the initial condition is thought to be a very small region, then the evolution is not deterministic. Lyapunov exponents can help, as a connexion between the two worlds. An initial condition that is a region could be thought of as an (uncountable) set of points. Choose two points inside that region, and therefore two deterministic trajectories, from the point of view of classic geometry: positive Lyapunov exponents mean that the trajectories separate very quickly and will quickly be separated by a larger distance than the initial region. That's the reason for the unpredictability from the point of view of classical mechanics, and since all one can know is that the initial condition is a region, that's a true indeterminism from the point of view of our mathematical formalism.

It should be useful to spell out in more details our reasoning: The peculiarity of such a system (Bunimovich billard) is that it is chaotic, but strictly and strongly deterministic. We consider it as a deterministic system because two different initial conditions lead to different evolutions in the future, but two identical initial positions lead to an identical evolution. If we consider regions as an initial condition, then it is not any more possible to consider the system as a deterministic one. In fact, consider a given region and two points inside that region: If two points are "inside the region" they are blurred and undistinguishable. Under the chaotic dynamic they will diverge very fast and become distinguishable. Then the system will no more be considered deterministic: An initial position such as "particle 1 in region 1 at time $t_{1}$ " can evolve toward the future in many different ways (at time $t_{2}$ the particle could be roughly everywhere if $t_{2}$ is far enough from $t_{1}$ ). The reason of this strange dependence on mathematics is that the meaning of 'identical' when speaking of identical initial condition depends on our mathematical model. In fact, 'identical' means 'situated in the same Euclidean point'. Therefore when we use 'identical' in describing chaotic systems, we introduce an artifice of mathematical language into our physical system: since the notion of determinism rely on the possibility of setting two 'identical systems' on an 'identical initial condition', changing the meaning of 'identical' (due to the change of the mathematical formalism), influence our belief in 'deterministic' chaos. Obviously determinism is ontologically true or false, but the solely reason to believe in the first or the second claim depends on the mathematical formalism we choose.

The choice of a different axiomatic for geometry changes our philosophical claims about the determinism or indeterminism of a physical theory. And what's even more 
important, the choice of a different axiomatic does not change our ability to describe the world. The deterministic trajectories of the pointillist and the indeterministic behavior of the point-free geometer are strictly equivalent in their ability to predict or explain the empirical observations. Therefore, there is no possible discrimination based on empirical grounds, no way to say "the point-free is right" or "the pointfree geometry agrees much better with observations", as we could say in the case of Kepler and Tycho. Two mathematical descriptions are possible, and there is no way of distinguishing which one is better. It does not even make sense to consider that a mathematical description is "better" than another, if our criterion has to rely on observation.

Concerning observation, as in the theory-laden case, adherents of two mathematical formalisms will not observe the same thing; they will have a different representation of reality, and therefore they will have a different way of conceptualize sensorial experience; but contrary to the theory-ladeness case, none of the adherents will find an empirical argument to win the battle. There is no such argument: there is no possible observation of a pure mathematical object. Nonetheless, mathematical objects guide our propositions about observation (e.g. telling to our students that we are studying deterministic chaos, or that a box at rest on the table is attracted by the earth, but it is also pushed by the reaction force, ...), and, therefore, observation is mathematically-laden.

\section{Conclusions}

We believe that our conclusions are relevant for philosophers of science, even though they don't affect the work of empirical scientists. An empirical scientist could use any mathematical instrument, and the results he obtains would remain unchanged. On the other side, our argument should sound as an alert to philosophers of science or to anyone concerned with foundations of sciences: we showed that our choice of a mathematical structure is not neutral; we choose because of esthetic, social or intellectual preference. We then draw conclusions about determinism, or the interpretation of quantum mechanics, or the existence of forces. But those conclusions are not solely motivated by our observations of the world, as we often believe: they are motivated by our choice of a mathematical structure; as a matter of fact it is not possible to clearly separate the mathematical conceptualization and the bare facts (as it is not possible in the theory-ladeness case). Each time we start interpreting, we should care about the background ontology implied by the mathematical structure we are using. Our case study is emblematic: we always speak of deterministic chaos, but the motivation is not empirically based; it comes from our choice of a mathematical structure. The mathematical structures used in the description of the world 
has an impact on its interpretation: For instance, because of a certain mathematical structure we pretend that the physical ontology of our world is deterministic.

That the world is deterministic is possibly a justified claim, but it should be carefully analyzed: it is far from being gratuitous. A justification based on the form of the equations is certainly not enough. Mathematics is maybe the most neutral language that we have, but it is not theory-neutral.

The mathematical-ladenness of observation implies that our philosophical interpretation is often related to the mathematical apparatus we use, instead of the observations and measurements we can perform. Since we cannot clearly evaluate the impact of using a given mathematical structure, there isn't any clear limit to the impact that the mathematical-ladenness could have on our best theories. ${ }^{9}$

\section{References}

Bramè, M. V. 2006. La metafisica di Whitehead nelle formule di Whitehead. Istituto di filosofia Arturo Massolo.

Bricmont, J. 1995. Science of chaos or chaos in science? Annals of the New York Academy of Sciences 775(1): 131-75.

Butterfield, J. 2006. Against Pointillisme about Mechanics. British Journal for the Philosophy of Science 57(4): 709-53.

URL: http://dx.doi.org/10.1093/bjps/ax1026

Earman, J. 1986. A primer on determinism. Dordrecht: D. Reidel publishing company. 1989. World Enough and Spacetime: Absolute and Relational Theories of Motion. Boston: MIT Press.

Esfeld, M. 2009. Philosophie des sciences. Lausanne: Presses polytechniques et universitaires romandes.

Friedman, M. 1983. Foundations of Space-Time Theories: Relativistic Physics and Philosophy of Science. Princeton: Princeton University Press.

Frigg, R. 2008, A field guide to recent work on the foundations of statistical mechanics. The Ashgate companion to contemporary philosophy of physics. London: Ashgate, pp.99-196.

Gerla, G. 1990. Pointless metric spaces. The Journal of Symbolic Logic 55(1): 207-19. URL: http://www.jstor.org/stable/2274963

Hanson, N. R. 1958. Patterns of discovery. Cambridge: Cambridge University Press.

Israel, G. 1992. L'histoire du principe du déterminisme et ses rencontres avec les mathématiques. Chaos et déterminisme. Paris: Editions du Seuil, pp.249-73.

. 1993. The two path of the mathematization of the social and economic sciences. Physis 30: 27-78.

-1996. La mathématisation du réel. Paris: Seuil.

Israel, G.; Ingrao, B. 1990. The invisible hand, economic equilibrium in the history of science. Cambridge, MA: MIT Press.

Jones, R. 1991. Realism about what? Philosophy of Science 58(2): 185-202.

Ladyman, J. 1998. What is structural realism? Studies In History and Philosophy of Science, Part A 29(3): 409-24.

Principia 17(1): 165-181 (2013). 
Madrid-Casado, C. M. 2008. A brief history of the mathematical equivalence between the two quantum mechanics. Latin-American Journal of Physics Education 2(2): 73-6.

- 2011. Sr. realista estructural, tenemos un problema: La carga ontológica de las matemáticas. Principia 14(2): 201-9.

Müller, T. M. 2006. La relatività di Whitehead, il sipario che non cala. In: M. V. Bramè (ed.), Il principio di relatività. Milano: Ed. Melquiades, pp.59-69. Saggio introduttivo a 'Il principio di relatività' di A.N. Whitehead.

Muller, F. 1997a. The equivalence myth of quantum mechanics - part 1. Studies In History and Philosophy of Science Part B: Studies In History and Philosophy of Modern Physics 28(1): 35-61.

Muller, F: 1997b. The equivalence myth of quantum mechanics - part 2. Studies In History and Philosophy of Science Part B: Studies In History and Philosophy of Modern Physics 28(2): 219-47.

- 1999. The equivalence myth of quantum mechanics - Addendum. Studies In History and Philosophy of Science Part B: Studies In History and Philosophy of Modern Physics 30(4): 543-5.

Norton, J. 2008. The dome: An unexpectedly simple failure of determinism. Philosophy of Science 75(5): 786-98.

Pecoraro, A.; Gerla, G. 2006. Formalizzazione della geometria senza punti di A.N. Whitehead. Master's thesis, Università degli studi di Salerno.

Prigogine, I.; Stengers, I. 1988. Entre le temps et l'éternité. Paris: Fayard.

Sellars, W. 1997 [1956]. Empiricism and the philosophy of mind. Cambridge, MA: Harvard University Press.

Suppes, P. 1993. The transcendental character of determinism. Midwest Studies in Philosophy 18(1): 242-57.

Werndl, C. 2009. Are deterministic descriptions and indeterministic descriptions observationally equivalent? Studies in History and Philosophy of Science Part B: Studies in History and Philosophy of Modern Physics 40(3): 232-42.

Werndl, C. 2013. On choosing between deterministic and indeterministic models: underdetermination and indirect evidence. Synthese 190(12): 2243-65.

URL: http://dx.doi.org/10.1007/s11229-011-9966-9

Whitehead, A. 1929. Process and reality. Cambridge: Cambridge University Press.

Thomas Michael Müller Centre Walras-Pareto d'études interdisciplinaires de la pensée économique et politique - IEPI

Geopolis - Université de Lausanne

1015 Lausanne

SWITZERLAND

thomas.muller.2@unil.ch

Resumo. Neste artigo, defenderemos a afirmação epistemológica de que a observação é determinada por matemática. Uma tese bem conhecida na filosofia da ciência é a de que a observação é determinada por teoria. A afirmação de que a observação é determinada por matemática pode ser justificada de maneira análoga. A primeira parte do artigo concentra-se

Principia 17(1): 165-181 (2013). 
na definição de determinação por matemática e suas relações com os bem conhecidos problemas de determinação por teoria. A segunda parte do artigo apresenta alguns exemplos específicos e delineia as consequências e dificuldades desse limite epistemológico. Finalmente, um contexto específico para esse problema é discutido em detalhe: analisaremos a questão do caos determinístico como um exemplo paradigmático de determinação por matemática e mostramos que a natureza determinística ou indeterminística do caos é fortemente ligada à escolha de uma descrição matemática particular.

Palavras-chave: Epistemologia; determinação por teoria; determinação por matemática; determinismo; caos determinístico.

\section{Notes}

${ }^{1}$ It is somehow dangerous to use the term theory as a synonymous of system of beliefs, a generic and vague notion. Despite the fact that a system of beliefs is a lousy defined concept, we prefer to avoid any richer definition. In our sense of theory/system of beliefs, no requirement of objectivity, or of realism is necessary. The theory of planetary motion, the theory of evolution, the astrological beliefs of ancient civilizations or any other inter-depending set of beliefs used as a guide for reasoning should be considered as a theory/system of beliefs. As a general guide to the rest of the article, we would use system of beliefs, when speaking in general, and we would prefer the term theory, when discussing a system of beliefs which has a (vindicated) scientific value, i.e. which is actually considered as a scientific theory. We will, for example, speak of a system of beliefs for the Aristotelian theory of motion, but we will speak of the theory of general relativity. This choice is only a conventional one, that depends on our opinion concerning scientific realism. In this article we will not enter in this dispute concerning scientific realism.

${ }^{2}$ We mean by observation an act of recording of a stimulus and of conceptualization of this stimulus. For example, when we switch on the camera of our computer, the machine will register what happens in front of the screen, but we will not call this an observation. An observation is in some sense a conscious perception; a computer can register a stimulus, but conceptualization is absent.

${ }^{3}$ Of course, not every discussion of this kind is entirely a matter of mathematical language, and we do not claim that any discussion of this sort is meaningless because of mathematically-ladeness.

${ }^{4}$ It has been suggested to us that other criteria such as computational simplicity, conceptual simplicity, the extension of the domain of application, etc could be used to separate two mathematical structures which are empirically equivalent. We personally think that conceptual simplicity is subjective. Computational simplicity and the extension of the domain of application could be used, but they are an epistemic criterion, not an ontological one. They will depend on the specific problem to be solved.

${ }^{5}$ A gas of $\mathrm{N}$ rigid balls in a square box has been proved to be ergodic for $N \leqslant 4$, but a general proof for any $\mathrm{N}$ is still missing. Nonetheless the system is (at least) chaotic. (Frigg 2008)

${ }^{6}$ For such a claim to be defendable, the reader should accept that a physical measurement could in principle be as precise as we want (no quantum uncertainty) but it can't be infinitely precise. I.e. one can improve as much as he wants the measurement he's doing, but he will

Principia 17(1): 165-181 (2013). 
always have a measurement error. An infinite precision in measurement simply does not make sense.

${ }^{7}$ Nonetheless we have a guide for intuition in the Whitehead-Gerla pointless geometry; regions are portions of space that can be occupied by physical objects, i.e. they have as many dimensions as the space in which we work, and they are a connected part of that space.

${ }^{8}$ We claim that this is the case if we use our example of regions as primitives. Other examples of stochastic models (Werndl 2009) have been provided in which this statement is false.

${ }^{9}$ My thanks to Laurent Cordonier, Giorgio Israel, Philippe-André Martin, Roberto Baranzini, Jan Lacki, Henri Volken, Michael Esfeld, Amanar Akhabbar, Raphael Sandoz, Nicolas Brisset, Olivier Jorand, Maxime Desmarais-Tremblay, Antoine Missemer, Antonio Vassallo, Tim Raez, Laura Croci-Rockinger, Jerome Meizoz and two anonymous reviewers of Principia for valuable comments to improve this paper.

Principia 17(1): 165-181 (2013). 